\title{
Jort (Calvados) « La Carrière Macé » : réexamen des données et nouvelles interprétations
}

Jort (Calvados) "La Carrière Macé" reexamining the data with new

interpretations

Jort (Calvados) "La Carrière Macé": revisión de la información y las nuevas interpretaciones

Jort (Calvados, Frankreich) „La Carrière Macé" Nachuntersuchung der alten Grabungsfunde und neue Interpretationen

\section{François Charraud}

\section{OpenEdition}

Journals

Édition électronique

URL : https://journals.openedition.org/rao/2892

DOI : $10.4000 /$ rao.2892

ISBN : 978-2-7535-5014-8

ISSN : $1775-3732$

\section{Éditeur}

Presses universitaires de Rennes

\section{Édition imprimée}

Date de publication : 31 décembre 2015

Pagination : 51-58

ISBN : 978-2-7535-5012-4

ISSN : 0767-709X

Référence électronique

François Charraud, « Jort (Calvados) «La Carrière Macé » : réexamen des données et nouvelles interprétations », Revue archéologique de l'Ouest [En ligne], 32 | 2015, mis en ligne le 28 avril 2018, consulté le 22 août 2022. URL : http://journals.openedition.org/rao/2892 ; DOI : https://doi.org/ $10.4000 /$ rao.2892 


\title{
Jort (Calvados) "La Carrière Macé » : réexamen des données et nouvelles interprétations
}

\author{
Jort (Calvados) "La Carrière Macé" Reexamining the Data with New Interpretations
}

\author{
François Charraud ${ }^{a}$
}

Résumé : Un réexamen récent du mobilier néolithique provenant de la fouille ancienne de Jort (Calvados) «La Carrière Macé » a permis de supposer que celui-ci était hétérogène. Ce constat a motivé une nouvelle étude de toutes les données disponibles sur ce site. Il s'avère que peu de structures relevées durant les années 1960 peuvent être datées car peu d'informations subsistent à leur sujet. L'essentiel du mobilier archéologique néolithique provient d'une grande fosse à la fonction et datation indéterminées. En se fondant sur la comparaison avec des exemples régionaux ou interrégionaux, on peut l'interpréter soit comme le sommet d'un puits d'extraction de silex, soit comme un four à chaux antique. Dans tous les cas l'hétérogénéité du mobilier néolithique est certaine et il serait approprié de l'écarter des réflexions sur la caractérisation de la culture matérielle du Néolithique ancien.

\begin{abstract}
A recent review of Neolithic items from the old archaeological excavation of Jort (Calvados) "Career Mace" assumed that they were heterogeneous. This has motivated a new study of all the available data from the site. Few of the features identified during the 1960 s can be dated because so little information exists about them. Most of the Neolithic finds are from a large pit, the function and date of which are not known. Based on a comparison with regional or interregional examples, the feature seems to be either the top of a flint mine or an ancient lime kiln. In all cases the heterogeneity of the Neolithic finds means that they are to be excluded from all debate on the characterization of Neolithic material culture.
\end{abstract}

Mots clés : Néolithique, Antiquité, Normandie, mine, silex, four à chaux.

Keywords: Neolithic, Antiquity, Normandy, mine, flint, lime kiln.

Le site de Jort, fouillé dans les années 1960, est resté pendant longtemps le seul site attribué au Néolithique ancien en Basse-Normandie. À plus forte raison, il constituait jusque dans les années 1990 le témoin le plus occidental de la diffusion du courant de néolithisation danubien, aux marges du Bassin parisien. Documenté par quelques notes succinctes (Bender, 1967; Dastugue, 1969), puis cité dans la thèse de C. Constantin (1985), le site n’a été publié qu'en 1992 par une équipe d'archéologues réalisant une synthèse sur la Normandie (Chancerel et al., 1992).
Il a depuis été cité dans plusieurs travaux de synthèse sans qu'aucun réexamen critique du contexte de découverte ne soit réalisé. Seul le mobilier céramique a été réétudié dans un travail universitaire (Gâche, 2007).

À l'occasion d'un nouvel examen réalisé pour les besoins d'une thèse (Charraud, 2013), plusieurs indices nous ont fait douter de l'homogénéité du mobilier de Jort. Ces questions ont motivé un réexamen complet du contexte de découverte et des archives de la fouille, avec l'aide de L. Dédouit et A. Ropars (SRA de Basse-Normandie).

${ }^{a}$ CEPAM - UMR 7264 CNRS / Université Nice Sophia Antipolis - Pôle universitaire de Saint-Jean-d'Angély 3 - 4 avenue des Diables-bleus - 06357 NICE Cedex 4. (fcharraud@gmail.com) 


\section{Contexte de découverte des vestiges}

Jort se trouve sur le flanc sud de la vallée de la Dives, à $13 \mathrm{~km}$ au nord-ouest de Falaise (fig. 1), à proximité du Douit du Houle, un petit affluent de la Dives, proche de la confluence. Cette zone est riche en occupations (ou indices) d'habitats ou de mines néolithiques (Dupuis et al., 2006; Charraud, 2013). Dans les environs du site ont été découverts en surface des vestiges du Paléolithique moyen (débitage Levallois), du Néolithique (lames, déchets de façonnage de haches), et de l'Antiquité (céramiques, terres cuites architecturales).

La fouille a révélé sept fosses attribuées au Néolithique, réparties sur un hectare et découvertes essentiellement en coupe dans le front de la carrière (fig. 2). Cependant, le mobilier provient de la seule structure fouillée (fosse 3 : fig. 3), et rien de permet d'affirmer que les autres fosses appartiennent à la même occupation : elles ne sont pas datées. On peut distinguer trois ensembles de fosses (fig. 2) qui ne sont pas documentées par des dessins précis.

- Les fosses 1, 2 et 3 sont de grandes fosses, profondes et caractérisées par des remplissages stratifiés alternant horizons sableux et charbonneux. Les fosses 1 et 2 ont été découvertes en front de carrière et relevées par B. Edeine en 1960, mais n'ont pas été fouillées. La fosse 3 est la seule fouillée entre 1961 et 1967 par M. Tieghem et son équipe.
- Les fosses 4, 5, 6 et 7 sont de petites dimensions, peu profondes et ovales. Elles ont été découvertes par M. Tieghem entre 1968 et 1970 . Les archives de fouille témoignent que ces structures ont été intégralement fouillées, mais il n'en subsiste aucun mobilier.

- Plusieurs tronçons (4 ou 5) de fossés gallo-romains ont également été fouillés par M. Tieghem entre 1968 et 1970, mais aucun plan précis n’en subsiste.

\section{UNE STRUCTURE INEXPLIQUÉE}

L'essentiel du mobilier archéologique provient de la fosse 3 (fig. 3), fouillée par moitié, l'autre moitié ayant été détruite par l'exploitation de la carrière. Aucun relevé en plan n'a été conservé. Dans les carnets de fouille de M. Tieghem, elle est décrite comme une structure ovale de 2,20 m par $1,50 \mathrm{~m}$, profonde de $3 \mathrm{~m}$. Son profil est tronconique évasé en son sommet. Ensuite, les parois fortement rubéfiées plongent verticalement, décrivant une forme cylindrique, jusqu'au fond de la structure, très plat. Les niveaux de remplissage montrent des épisodes successifs de rebouchage :

- la partie supérieure de la fosse (fig. 3 : couche A) est caractérisée par un sédiment noirâtre qui comporte beaucoup de silex et quelques tessons; elle est attribuée
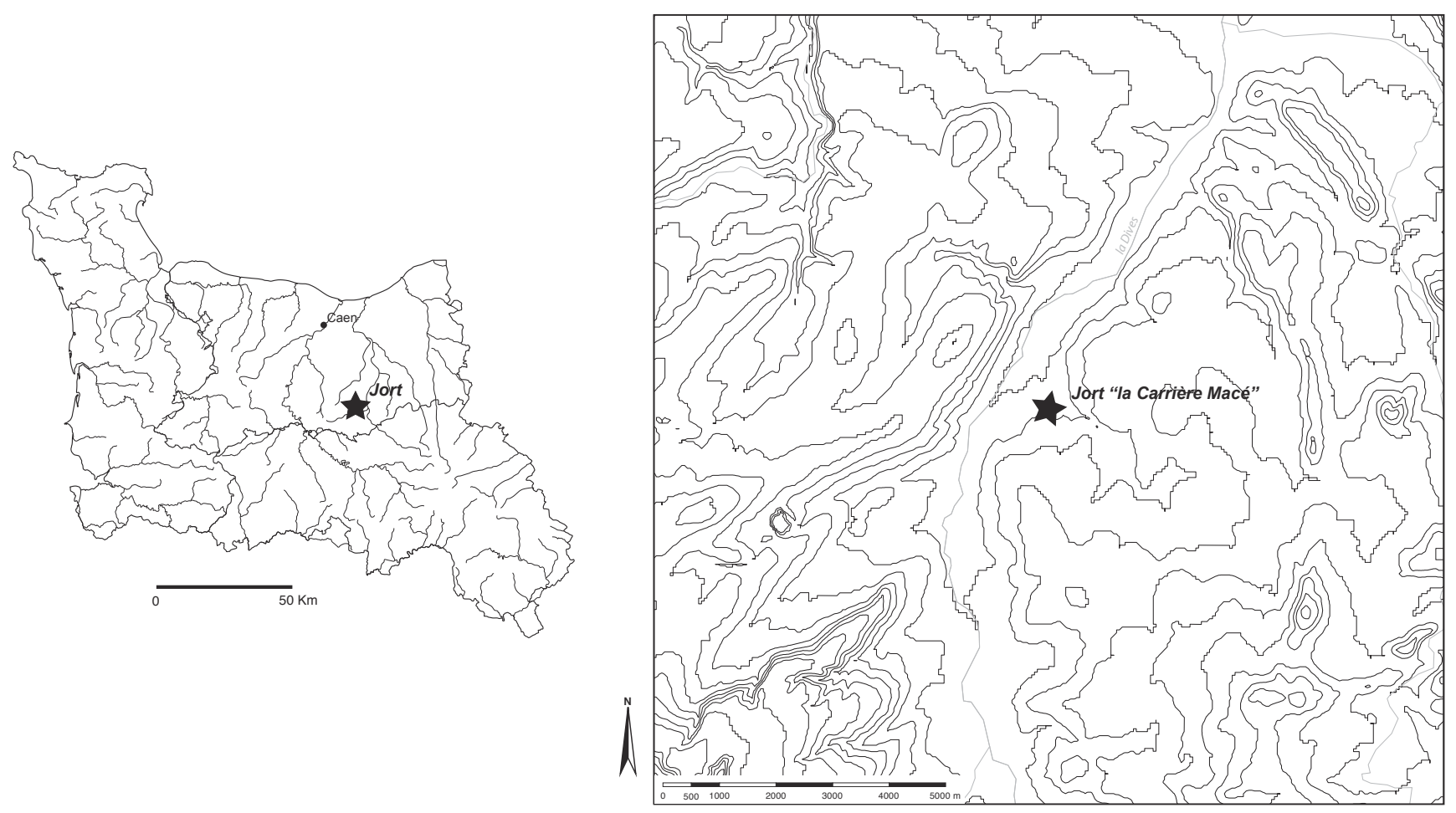

Figure 1 : Localisation du site de Jort et contexte topographique.

Figure 1: Location of the Jort site and its topographical context. 


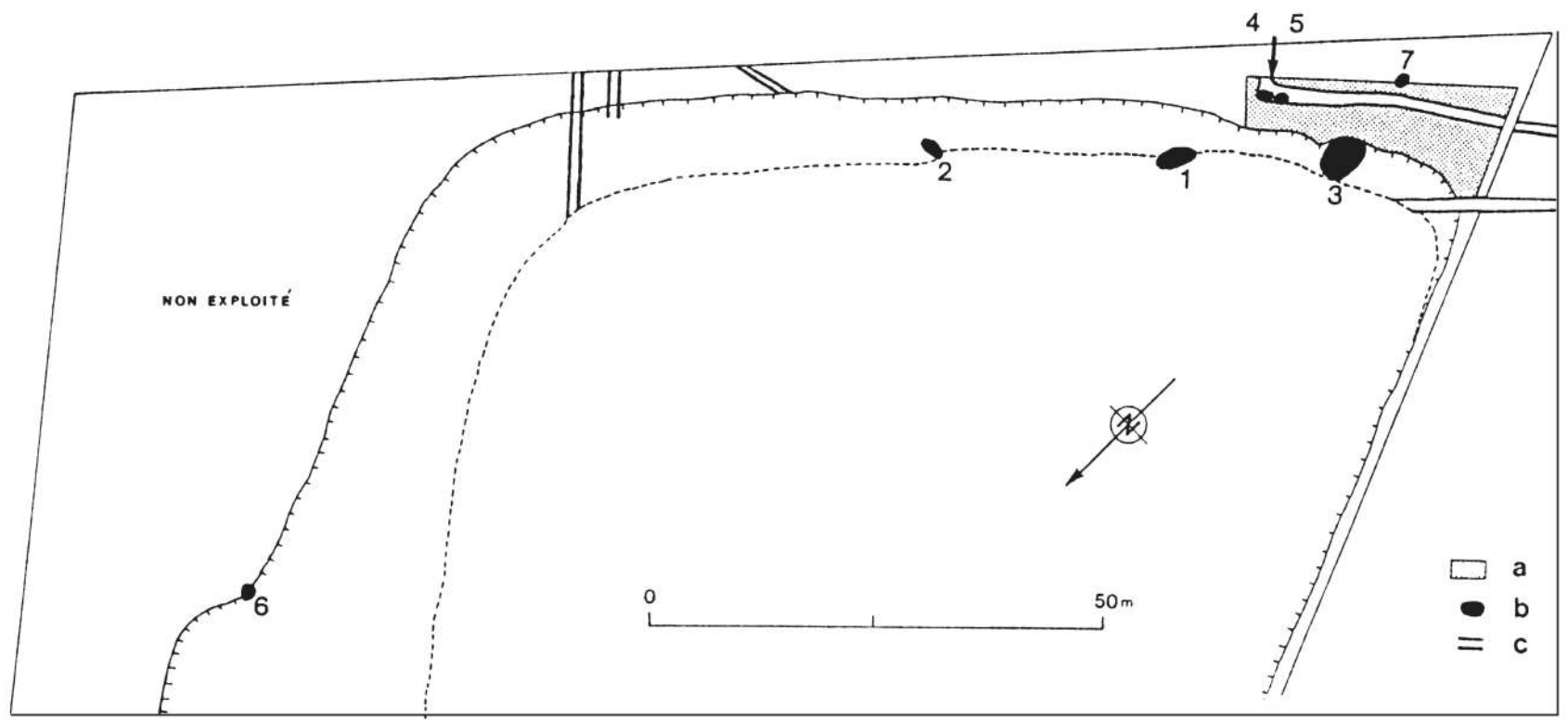

Figure 2 : Plan de la carrière et des structures fouillées par B. Edeine et M. Tieghem entre 1960 et 1970. a : zone décapée lors des fouilles; $\mathrm{b}$ : fosses archéologiques; $\mathrm{c}$ : fossés galloromains.

Figure 2: Map of the quarry pit and the features excavated by Edeine and M. Tieghem between 1960 and 1970. a: excavated zone; b: archaeological pits; c: Gallo-roman ditches.

Figure 3 : Coupe stratigraphique de la structure 3 telle que relevée par $\mathrm{M}$. Tieghem (d'après Dastugue, 1969, p. 421).

Figure 3:

Stratgraphical section of structure 3 as drawn by $M$. Tieghem.

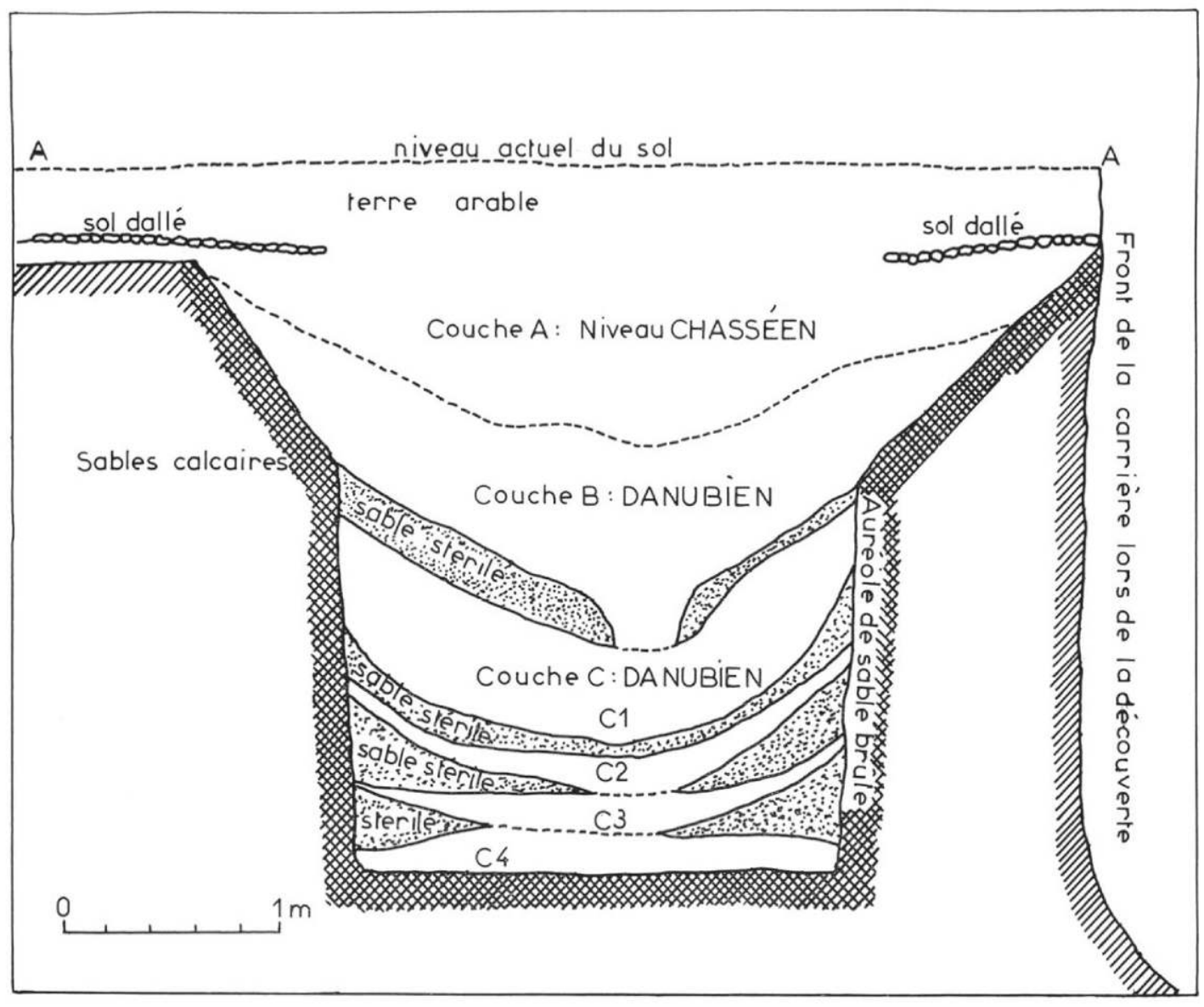


au "Chasséen du Bassin parisien " à la suite de la fouille (Dastugue, 1969 : p. 421).

- la seconde partie du remplissage (fig. 3 : couche B) est semblable à la précédente, quoique moins sombre, et contient davantage de mobilier; ce niveau et les horizons sous-jacents sont attribués au "Danubien récent, groupe de Cerny» (ibid. p. 421) par les fouilleurs.

- la troisième partie du remplissage (fig. 3 : couches $\mathrm{C} 1$ à C4) est décrite comme une alternance de couches noires consistant en de "nombreux éléments sableux mélangés à des cendres de foyer " et de couches de "sables stériles à l'état de chaux durcie" (Dastugue, 1969 : p. 422).

On peut également mentionner une "racine de vieux sol " (ibid. p. 421 et fig. 3) surmontée d'un "sol dallé", tel que décrit par les auteurs de la fouille et repris dans la publication (Chancerel et al., 1992). Toutefois, les photographies prises lors de la fouille montrent qu'il s'agirait plutôt de la semelle de labour damée par les travaux agricoles, surmontant un horizon argileux à plaquettes calcaires. Le tout ressemble fortement à un niveau colluvial mince surmontant le substrat.

La fosse 3 a d'abord été interprétée comme un "fond de cabane " et "foyer d'habitat" (Dastugue, 1969), puis comme un silo (Chancerel et al., 1992). Cependant, les archives de la fouille empêchent de souscrire à ces propositions. Même en supposant une dégradation prononcée de la structure lors de son utilisation, un silo si imposant, à ouverture évasée ou non, ne trouve aucun élément de comparaison néolithique. De plus une ouverture large ne facilite pas une fermeture hermétique de la structure et la nature très perméable de l'encaissant (calcaire déstructuré sableux) se prête mal à cette fonction.

\section{Problèmes D'attribution CHRONOCULTURELLE}

Par ailleurs, la distinction des deux niveaux de remplissage, l'un "danubien " et l'autre " chasséen ", tels que décrits par M. Tieghem et J. Dastugue (1969), n’a pas été relayée dans la publication de 1992 (ibid.). De plus, le conditionnement actuel du mobilier dans les réserves du SRA de Basse-Normandie ne semble pas respecter le conditionnement initial : les différents lots ont été mélangés au gré de manipulations successives. Les différents types de mobilier ne peuvent donc pas être associés à des horizons stratigraphiques particuliers.

D'une part, le mobilier comprend des caractères qui se rapportent au Villeneuve-Saint-Germain :

- les fragments de parures en schiste (Fromont, 2012);
- les céramiques à forme globulaire et à décors au peigne (Gâche, 2007);

- un fragment de lame en silex tertiaire du Bassin parisien (Augereau et Bostyn, 1997);

- les nombreux outils sur lame en silex du Cinglais, typologiquement caractéristiques de cette culture. Ces outils renvoient à un large panel d'outils domestiques (Charraud, 2013).

Mais des éléments plus récents sont également présents :

- certains éléments lithiques non caractéristiques sont peu altérés, comparés à l'outillage typologiquement caractéristique du Néolithique ancien (Charraud, 2013);

- un vase à profil en $S$ non décoré renvoie au Néolithique moyen 2 (Gâche, 2007);

- une unique datation par le radiocarbone réalisée sur un charbon de bois de la fosse 3 donne un résultat postérieur au VSG : 4400-4350 BC Cal. (probabilité 95,4 \%; Chancerel et al., 1992).

Par conséquent, l'attribution chrono-culturelle de cet ensemble au Villeneuve-Saint-Germain n'est pas complètement recevable.

Ces indices suggèrent que le creusement de la fosse 3 est plus récent qu'une partie du mobilier qu'elle contient : il pourrait dater du Néolithique moyen si l'on considère les éléments significatifs les plus récents. Le mobilier caractéristique du Néolithique ancien, apporté dans les terres de comblement de la fosse, proviendrait alors d'une occupation antérieure. Les autres structures relevées par M. Tieghem pourraient d'ailleurs s'y rapporter. Mais en partant du même raisonnement, le creusement peut également être postérieur au Néolithique moyen.

Ce sont donc à la fois la datation et la fonction de cette structure qui sont inexpliquées.

\section{Première hypothèse : UNe STRUCTURE D'EXTRACTION DE SILEX NÉOLITHIQUE?}

Quelques indices suggèrent que cette fosse pourrait être le sommet d'un puits d'extraction de silex. Il existe une probabilité que le fond de la structure n'ait pas été atteint par les fouilleurs : quelques photographies d'archives montrent que la fouille a été réalisée en coupe, à l'aide d'une pelle mécanique et d'un godet court à dents qui a laissé la coupe dans un état illisible (fig. $4, \mathrm{n}^{\circ} 1$ ). Un rafraîchissement de cette coupe a ensuite été effectué manuellement. Pour être à la hauteur du sommet du remplissage, les fouilleurs sont debout sur un cône de déblais appuyé sur la base de la coupe (fig. $4, n^{\circ} 3$ ). Dans ces conditions, le fond de la fosse apparaît au niveau des pieds des fouilleurs, il est illisible, et son fond plat peut être dû à un effet de fouille (fig. 4, n 3 ). 
La comparaison avec les puits fouillés sur les minières de silex de Ri « le Fresne " (Orne : Marcigny et al., 2011) ou d'Espins " Foupendant " (Calvados: Charraud et al., 2009) montre qu'en cas de sédiments meubles, les premiers niveaux de remplissage sont souvent difficiles à distinguer de l'encaissant, même avec l'expérience de ce type de contextes. Il n'est donc pas exclu que la fosse 3 de Jort soit le sommet d'un puits d'extraction de silex, dont les fouilleurs n'auraient pas distingué le fond, à une époque où ce type de sites était peu documenté dans la région.

Un sondage géologique permettrait de déterminer si du silex de bonne qualité est présent dans l'environnement, ce qui étayerait cette idée. Par ailleurs, on peut supposer que des structures semblables, non fouillées, sont conservées à proximité, ce que suggère une photographie prise par J. Dastugue en avril 1967 (fig. 4, n 4).

Mais dans ce cas, rien n'explique la forte rubéfaction des parois de la structure qui suggère une structure de combustion...

\section{SECONDE HYPOTHÈSE : UN FOUR À CHAUX ANTIQUE?}

Ceci nous amène à l'hypothèse, selon nous plus probante, que cette fosse est une structure de combustion, mais aucun élément de comparaison néolithique ou protohistorique n’a
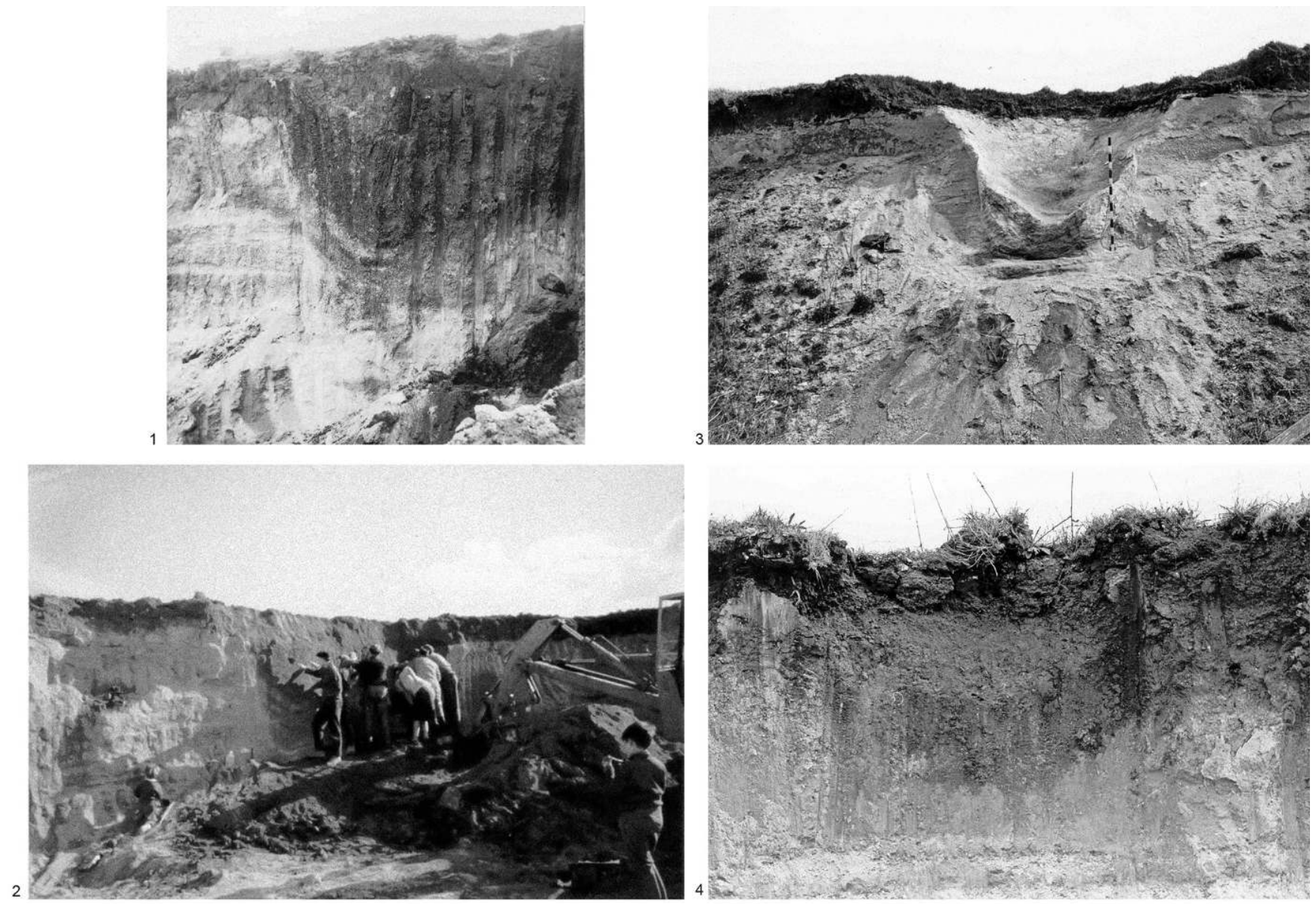

Figure 4 : Photographies de la structure 3, prises lors des investigations successives (source : archives du SRA de Basse-Normandie, documents fournis par L. Dédouit, MCC). 1 : cliché daté du 13 mars 1960, signé E. L. (Edouard Lagnel ?), montrant l'état de la coupe de la fosse 3 après avivage réalisé au godet à dents. 2 : cliché de J. Dastugue, daté de mars 1967, légendé "État où nous avons trouvé la fouille de M. l'abbé Tieghem ». 3 : cliché de 1960 (auteur inconnu) montrant la fouille en coupe de la fosse 3, les fouilleurs juchés sur un cône de déblais. 4 : cliché de J. Dastugue montrant une fosse (ou sommet de fosse?) autre que la fosse 3, observée dans le front de la carrière. Figure 4: Photos of the structure 3 taken during the different investigations (source SRA de Basse-Normandie, documents provided by L. Dédouit, MCC). 1: photo dating from 13 March 1960, signed E. L. (Edouard Lagnel.), showing the state of the section of structure 3 after l'état de la coupe de la fosse 3 after "refreshening" using a denticulated bucket. 2: photo by J. Dastugue, dated to March 1967, with caption "State in which we found the excavation of M. l'abbé Tieghem". 3: photo from 1960 (unknown author) showing the excavation of pit 3's section, the diggers are standing on the spoil heap. 4: Photo by J. Dastugue showing a pit (the top?) other than pit 3, in the quarry face. 
pu être mis en évidence. C'est en fait l'alternance des horizons charbonneux et de "niveaux sableux à l'état de chaux durcie " décrits par les fouilleurs, qui suggèrent la chaîne opératoire de production de la chaux.

Les parois de la fosse, rubéfiées sur plusieurs centimètres, supposent une température importante, et une durée suffisante, comme le montrent des expérimentations menées à propos des structures de combustion du proche site de Condé-sur-Ifs « la Bruyère du Hamel » (Dron et al., 2010; Fromont, 1999). Partant de cette hypothèse, on trouve effectivement des exemples de fours à chaux de l'Antiquité ou du haut Moyen Âge, en France et en Allemagne, très comparables à la fosse 3 de Jort : un four à chaux de " la Montée de la Butte " à Lyon (v viècle après J.-C., fig. 5) montre une structure en creux d'un module proche associant un fond plat, une chambre de chauffe cylindrique et une ouverture évasée ovale, originellement coiffée d'une cheminée maçonnée (Motte et al., 2009). L'alternance de niveaux de chaux et de charbons de bois, comme à Jort, est caractéristique du remplissage de ce type de structure si elles n'ont pas été curées avant abandon (ibid. et fig. 5). Elle est liée à la technique d'enfournement des matériaux : le calcaire est enfourné en alternance avec des litages de combustible.
La situation topographique de la fosse 3 s'y prête également, car les fours à chaux gallo-romains sont souvent installés au pied d'une déclivité ou d'un talus : le creusement dans le sol favorise l'inertie thermique et facilite le chargement des matériaux par le haut (ibid.). Ils sont également souvent situés à proximité d'une carrière, pour faciliter d'approvisionnement en matériaux (Boudartchouk, 2003). Cela supposerait l'ancienneté de l'exploitation de la carrière Macé.

D’une manière générale, les fours à chaux gallo-romains sont de plan circulaire mais leurs dimensions sont extrêmement variables. Les plus gros ( $4 \mathrm{~m}$ de diamètre) sont $\mathrm{du}$ module de celui de Bourgheim dans le Haut-Rhin (II siècle après J.-C. : Pétry, 1982). De nombreux exemples existent également en Allemagne (Sölter, 1970).

Dans cette hypothèse, les aménagements liés à l'alimentation en combustible du four (couloir de chauffe ou fosse d'accès, foyer, alandier), se trouveraient dans la moitié détruite par le front de la carrière et la moitié fouillée de la fosse 3 serait ce qui reste de la sole et de la chambre de chauffe. Comme dans l'hypothèse du puits de mine, les vestiges néolithiques seraient en position remaniée dans les terres de comblement de la structure et proviendraient d'une occupation antérieure.

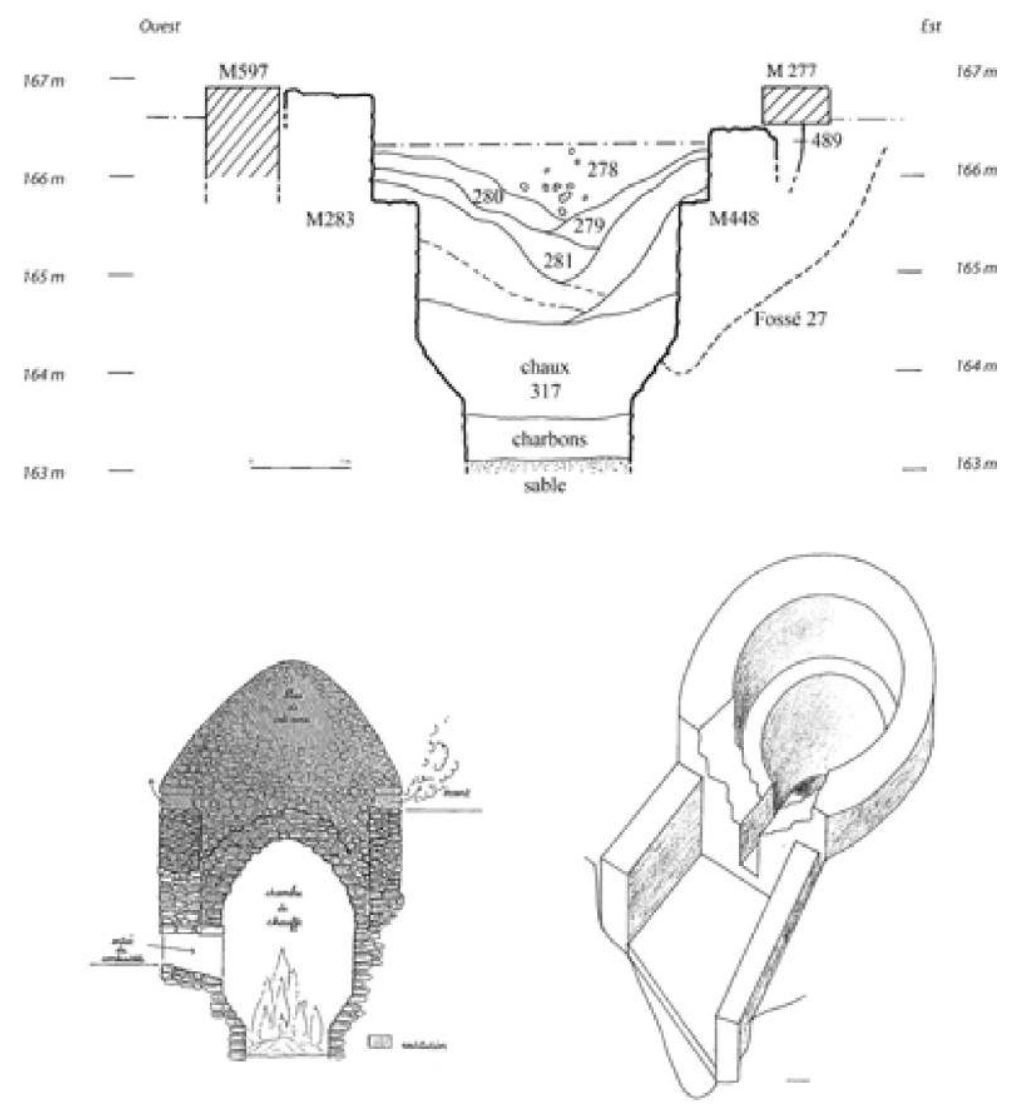

Figure 5 : Le four à chaux de " la Montée de la Butte " à Lyon ( $\mathrm{v}^{\mathrm{e}}$ siècle après J.-C.) : coupe stratigraphique et essais de restitution, d'après Motte et al., 2009, p. 348.

Figure 5: The lime kiln at "la Montée de la Butte", Lyon ( $5^{\text {th }}$ century $\left.A D\right)$ : section and restitution, after Motte et al., 2009, p. 348. 


\section{Conclusions}

Quelle que soit la fonction que l'on prêtera à la fosse 3, nous pouvons désormais affirmer que le mobilier qui en provient est hétérogène et qu'il ne peut pas être utilisé pour caractériser la culture matérielle du Néolithique ancien, comme il l'a été à plusieurs reprises.

Pour ce qui concerne la fonction et la datation de la fosse 3 , les deux interprétations proposées ici ne sont peut-être pas les seules possibles mais elle ne peut pas être antérieure au Néolithique moyen, ce qui entérine la discontinuité avec le mobilier VSG qu'elle contient. Qu'il s'agisse d'une mine de silex néolithique, d'un four à chaux gallo-romain, ou d'autre chose, il est donc certain que le mobilier du Néolithique ancien qui a été récolté lors de la fouille provient d'une occupation antérieure au creusement. La présence de céramiques et d'un panel d'outils diversifié signale sans doute une occupation domestique sur ce versant de la vallée de la Dives. Les conditions y sont en effet idéales, à proximité de placages limoneux fertiles, de cours d'eau abondants et de gîtes de silex de bonne qualité. L'imprécision de ce contexte ne permet donc pas d'interpréter les vestiges mobiliers dans un cadre archéologique significatif.

\section{Remerciements}

L'accès aux archives de fouille du SRA de Basse-Normandie a été possible grâce à l'aide et la collaboration de Cyrille Billard, Laure Dédouit, Anne Ropars.

\section{Bibliographie}

Augereau A. et Bostyn F., I997 - "Évolution des industries lithiques entre le Villeneuve-Saint-Germain et le Cerny dans la France du nord ", in Constantin C., Mordant D. et Simonin D. (dir.), La culture de Cerny. Nouvelle économie, nouvelle société au Néolithique, Actes du colloque international de Nemours 1994, Mémoire du musée de Préhistoire d'Île-deFrance, ${ }^{\circ} 6$, Paris, p. 25-38.

Bender M.L., 1967 - The Neolithic Cultures of North-West-France, Thèse de Doctorat, Londres, 743 p.

Boudartchouk J.-L., 2003 - «La villa rustique de Larajadé (Auch, Gers), un petit établissement rural aux portes d'Augusta Auscorum : approche archéologique ", Aquitania, t. 19, Bordeaux.

Chancerel A., Desloges J., Dron J.-L. et San Juan G., I 992 "Le début du Néolithique en Basse-Normandie ", in Le Roux C.-T. (dir.), Paysans et bâtisseurs : l'émergence du Néolithique atlantique et les origines du mégalithisme, Actes du $17^{e}$ colloque interrégional sur le Néolithique (Vannes, 29-31 octobre 1990), Revue archéologique de l'Ouest, suppl. n 5, Caen, p. 153-173.

Charraud F. avec la coll. de Desloges J., Corbin L., Coutard J.-P., Dron J.-L., Fromont A., Fromont N., Giazzon S., Ladjadj J., Thomas Y., Ropars A. et Savary X., 2009 L'acquisition et la transformation initiale du silex du Cinglais dans la Plaine de Caen à la Préhistoire récente, Rapport de prospection thématique, Service régional de l'Archéologie de BasseNormandie, Caen, 103 p.

Charraud F., 2013 - Espaces interculturels et évolution des systèmes techniques au Néolithique dans le Nord-Ouest de la France. Productions, usages et circulation des outillages en silex jurassiques de Normandie, Thèse de doctorat de l'université Nice-Sophia Antipolis, 2 vol., Nice, 480 et 538 p.

Constantin C., I985 - Fin du Rubané, céramique du Limbourg et post-Rubané. Le Néolithique le plus ancien en Bassin parisien et en Hainaut, BAR international Series, 273, 2 vol., Oxford, 356 et 294 p.

Dastugue J., I969 - " Informations archéologiques. Circonscriptions de Haute et Basse-Normandie ", Gallia Préhistoire, no 12-2, Paris, CNRS, p. 417-437.

Dron J.-L., Germain-Vallee C., Clement-Sauleau S., Gache D., Charraud F. et Fromont N., 20 io - « La Bruyère du Hamel à Condé-sur-Ifs (Calvados). Un site entre Néolithique ancien et Néolithique moyen ", in BILlard C. et Legris M. (dir.), Premiers Néolithiques de l'Ouest. Cultures, réseaux, échanges des premières sociétés néolithiques à leur expansion, Colloque interrégional sur le Néolithique, Le Havre, 9-10 novembre 2007, Rennes, Presses universitaires de Rennes, p. 163-179.

Dupuis C., Giazzon D. et Giazzon S., 2006 - Prospection diachronique dans la vallée du Laizon, in V. Juhel dir. Archéologie et prospection en Basse-Normandie, Mémoires de la Société des antiquaires de Normandie, t. 38, Caen, p. 204-208.

Fromont S., I 999 - Approche pétrographique et diffractométrique de pierres chauffées du site de Condé-sur-Ifs, Mémoire de maîtrise de l'université de Caen, Caen, n. p.

Fromont N., 2013 - Anneaux et cultures du Néolithique ancien. Production, circulation et utilisation entre massifs ardennais et armoricain, BAR International Series, 2499, Oxford, 686 p.

GÂCHE D., 2007 - Identité de la céramique du Néolithique ancien en Basse-Normandie et ses marges occidentales, Mémoire de Master 1 de l'université Rennes 2, Rennes, 118 p.

Marcigny C., Ghesquière E., Giazzon D., Tsobgou Ahoupe R., Charraud F., Juhel L. et Giazzon S., 20 i I - "The flint mine of $\mathrm{Ri}$ "Le Fresne" ", in Capote M., Consuegra S., Díaz del Río P., Terradas X. (dir.), Proceedings of the $2^{\text {nd }}$ International Conference of the UISPP Commission on Flint Mining in Pre-and Protohistoric Times (Madrid, 14-17 October 2009), B.A.R. International Series 2260, Oxford, p. 67-75. 
Motte S. et Blaizot F. avec la coll. de Bonnet C., Cecillon C, Orengo L., Plantevin C. et Vaireaux F., 2009 "Contribution à la topographie antique de Lugdunum : la porte monumentale et l'espace funéraire du site de la montée de la Butte", Revue archéologique de l'Est, t. 58, Dijon, p. 325-380.

Petry F., I 982 - "Informations archéologiques, circonscription d'Alsace », Gallia, t. 40, fasc. 2, éd. du CNRS, Paris, p. 347-371.
SÖLTER W., I97I - Römische Kalkbrenner im Rheinland. Rheinische Kunststätten Heft 490. Rheinischer Verein für Denkmalpflege und Landschaftsschutz, Düsseldorf, 32 p.

Zusammenfassung: Jort (Calvados, Frankreich) „La Carrière Macé" Nachuntersuchung der alten Grabungsfunde und neue Interpretationen - Eine jüngst durchgeführte erneute Untersuchung neolithischen Fundmaterials aus der alten Ausgrabung von Jort (Calvados, Normandie, Frankreich) „La Carrière Macé" hat zur Schlussfolgerung geführt, dass diese Funde heterogener Herkunft sind. Diese Festsetllung hat dazu motiviert, alle verfügbaren Materialen zu dieser Altgrabung zu sichten und neu zu bewerten. Dabei hat sich gezeigt, dass nur wenige der in den 1960er Jahren dokumentierten Befunde datiert werden können, da zu ihnen nur sehr wenige Informationen vorliegen. Der überwiegende Teil des archäologischen Fundmaterials neolithischer Zeitstellung stammt aus einer großen Grube, deren Funktion und Zeitstellung unbekannt sind. Stützt man sich auf einige regionale und interregionale Vergleiche, kann man den Grabungsbefund entweder als obere Schachtöffnung einer Flintmine oder als einen antiken Kalkbrennofen interpretieren. Insgesamt muss die Heterogenität des neolithischen Fundmaterials als gesichert angenommen werden. Es erscheint deshalb angezeigt, diese Funde nicht in weitere Überlegungen zu einer Charakterisierung der materiellen Kultur des frühen Neolithikums einzubeziehen.

Resumen: Jort (Calvados) "La Carrière Macé": revisión de la información y las nuevas interpretaciones - Una revisión reciente del material neolítico de la antigua excavación de Jort (Calvados) "La Carrière Macé" permitió suponer que éste era heterogéneo. Esta conclusión ha motivado la realización de un nuevo estudio de todos los datos disponibles a propósito de este sitio. Resulta que pocas estructuras identificadas durante la década de 1960 se pueden fechar porque poca información queda sobre ellas. La mayoría de los artefactos arqueológicos neoliticos procede de un gran hoyo de función y fecha indeterminadas. Basándose en la comparación con ejemplos regionales o interregionales, se puede interpretar sea como la punta de un pozo de extracción de silex sea como un antiguo horno de cal. En todos los casos, la heterogeneidad del material neolítico es cierta y sería apropiado despedirla de las reflexiones sobre la caracterización de la cultura material del Neolitico antiguo.

Schlüsselwörter: Neolithikum, Römische Zeit, Normandie, Flintbergwerk, Flint, Kalkofen.

Palabras clave: Neolitico, Edad antigua, Normandía, mina, sílex, horno de cal. 